\title{
CAG-repeat variant in the polymerase $\gamma$ gene and male infertility in the Chinese population: a meta-analysis
}

\author{
Shu-Yuan Liu ${ }^{1}$, Chang-Jun Zhang ${ }^{2}$, Hai-Ying Peng ${ }^{2}$, Yu-Feng Yao ${ }^{1}$, Lei Shi ${ }^{1}$, Jin-Bao Chen ${ }^{1}$, Ke-Qin Lin ${ }^{1}$, \\ Liang $\mathrm{Yu}^{1}, \mathrm{Li} \mathrm{Shi}^{1}$, Xiao-Qin Huang ${ }^{1}$, Hao Sun ${ }^{1}$ and Jia-You Chu ${ }^{1}$
}

Several studies have reported a relationship between the length of the CAG-repeat in the polymerase $\gamma(P O L G)$ gene and male infertility. However, other studies have not reproduced this result. In our study, the POLG-CAG-repeat length was analyzed in 535 healthy individuals from six Chinese Han populations living in different provinces. The frequencies of 10-CAG alleles and genotypes were high ( 97.38 and $94.13 \%$, respectively), with no significant difference among the six Chinese Han populations. Furthermore, we determined the distribution of the POLG-CAG-repeat in 150 infertile men and 126 fertile men. Our study suggested that the distributions of POLG-CAG-repeat alleles and genotypes were not significantly different between infertile ( 95.67 and $92.67 \%$, respectively) and fertile men (97.22 and $94.44 \%$, respectively). In a subsequent meta-analysis, combining our data with data from previous studies, a comparison of the CAG-repeat alleles in fertile versus infertile men showed no obvious risk for male infertility associated with any particular allele (pooled odds ratio $(\mathrm{OR})=0.94 ; 95 \%$ confidence interval $(\mathrm{Cl}): 0.60-1.48$ ). The significance level was not attained with any of the following genetic models: homozygote comparison (not $10 /$ not 10 versus $10 / 10$ : $\mathrm{OR}=1.34 ; 95 \% \mathrm{Cl}: 0.66-2.72$ ),

heterozygote comparison (10/not 10 versus 10/10: $\mathrm{OR}=1.04 ; 95 \% \mathrm{Cl}$ : $0.78-1.38$ ), dominant model comparison (not 10/not 10+10/ not 10 versus 10/10: $\mathrm{OR}=1.08 ; 95 \% \mathrm{Cl}$ : $0.79-1.47$ ) and recessive genetic comparison (not 10/not 10 versus 10/not 10+10/10: $\mathrm{OR}=1.31 ; 95 \% \mathrm{Cl}: 0.68-2.55)$. In conclusion, there is no significant difference of the frequencies of $P O L G$-CAG-repeat variants among six Chinese Han populations, and this polymorphism may not be associated with Chinese male infertility. On the basis of a meta-analysis, there is no obvious association between CAG-repeat variants of the POLG gene and male infertility.

Asian Journal of Andrology (2011) 13, 298-304; doi:10.1038/aja.2010.91; published online 22 November 2010

Keywords: case-control study; male infertility; meta-analysis; POLG-CAG-repeat

\section{INTRODUCTION}

Male infertility is a multifactorial syndrome encompassing a wide variety of disorders. Oligozoospermia, asthenozoospermia, teratozoospermia and azoospermia are the four main clinical types of infertility, and these account for $20-25 \%$ of cases. ${ }^{1}$ Researchers have proven a genetic contribution to infertility by demonstrating genetic influences on a variety of physiological processes, including hormonal homeostasis, spermatogenesis and sperm quality. ${ }^{2}$ Because spermatozoa contain a large number of mitochondria and mitochondria have an important role in the quality and quantity of spermatozoa by providing the energy needed to complete their functions, especially sperm motility, ${ }^{3}$ it is generally hypothesized that the accumulation of pathogenic mitochondrial DNA (mtDNA) influences the function of spermatozoa, including sperm motility. ${ }^{4}$ A series of studies have noted an association between alterations of mtDNA and sperm dysfunction. ${ }^{4}$

DNA polymerase $\gamma$ (POLG), which is encoded by the POLG gene, is the only known DNA polymerase for mtDNA replication and maintenance in human beings. The human POLG gene is located on 15q2415q26, spans 23 exons and includes a trinucleotide CAG-repeat region that encodes a polyglutamine stretch near the $\mathrm{N}$-terminus of the mature protein, downstream of the presumed mitochondrial targeting sequence..$^{5-7}$ It has been reported that human cDNA sequences contain 10 consecutive glutamines encoding CAG codons, followed by a single glutamine encoding a CAA codon and then two further CAG codons. ${ }^{8}$ Polyglutamine tracts can be sites for protein-protein interactions; altering the tract in POLG may result in suboptimal or improper mtDNA replication. ${ }^{5}$

Previous studies have shown that the common allele contains 10 repeats in exon 1 of the POLG gene in European populations $(88 \%)^{9}$ and northern Eurasian populations (88-96\%). ${ }^{10}$ More and more evidence has indicated that the length of the CAG-repeat may influence the function of spermatozoa, and several studies have confirmed that an alteration in the length of the CAG-repeat is associated with male infertility; ${ }^{9,11,12}$ however, some studies have failed to establish such an association. ${ }^{13-19}$ Because there are different distributions of the CAG-repeat length among healthy individuals from different ethnic groups, ${ }^{10,14,16}$ a determination of the distribution of CAG-repeat length is necessary before an association study between male infertility and CAG-repeat variants in the POLG gene can be carried out.

In this study, we investigated the distribution of CAG-repeat lengths in six Chinese Han populations living in six different provinces to establish the range of this trinucleotide CAG-repeat length

${ }_{1}^{1}$ Department of Medical Genetics, Institute of Medical Biology, Chinese Academy of Medical Sciences and Peking Union Medical College, Kunming 650118, China and ${ }^{2}$ Reproductive of Medical Research Centre of People's Hospital of Shiyan 442000, Shiyan, China Correspondence: Dr J Y Chu (Chujy08@gmail.com)

Received: 6 May 2010; Revised: 12 June 2010; Accepted: 13 July 2010; Published online: 22 November 2010 
polymorphism in individuals of Chinese Han ethnicity. Then, we studied the association between the asthenozoospermia and oligoasthenozoospermia types of male infertility in the Chinese population and CAG-repeat length variants within the POLG gene. In addition, we combined our data with data from previous studies in a carefully designed meta-analysis to study the association between CAG-repeat variants of the POLG gene and male infertility.

\section{MATERIALS AND METHODS}

\section{Study participants}

Healthy Chinese Han individuals. To establish the distribution of POLG-CAG alleles in a population of Chinese ethnicity, a total of 535 unrelated healthy Chinese Han people from six different provinces (Shandong, Gansu, Hunan, Yunnan, Guangxi and Guangdong provinces) were included in the study. Samples were collected from the project 'Establishment and preservation of permanent growing B cell line of ethnic groups of China', supported by the China Medical Board of New York Inc. (CMB 04-805).

Blood samples were obtained from these individuals with informed consent. Genomic DNA was extracted from peripheral lymphocytes by a standard hydroxybenzene-chloroform method.

Participants for case-control study. A total of 276 male participants attending the Reproductive Medical Research Centre of People's Hospital of Shiyan in Hubei Province for assisted reproductive therapy were recruited in this case-control study. All the participants were $\leqslant 40$ years of age.

Within the group of 276 participants, there were 150 infertile men. All infertile men were routinely screened by karyotyping and examinations of phenotype (physique, development of testes and penis) and hormonal status. A team of andrologists conducted detailed clinical investigations and recorded complete case histories (including pathology and therapy that may have influenced fertility, such as orchitis, injury to the testes, diabetes, tuberculosis, chronic respiratory diseases, pancreatic cystic fibrosis, adult mumps, neuromuscular symptoms and radiotherapy or chemotherapy for cancer), the fertility status of male relatives, history of diseases affecting fertility (such as urethral strictures, hypospadias, resection of the prostate, bladder neck surgery, vasoresection, inguinal hernia, hydrocele surgery, history of sympathetic surgery or other surgeries, urinary system infection, sexually transmitted diseases and epididymitis) and other factors that may cause male infertility (fever, smoking, exposure to toxins, alcoholism and drug addiction). Patients with chromosomal abnormalities and genital tract pathologies were excluded. All study participants underwent semen analysis according to the guidelines of the World Health Organization. ${ }^{20}$ Eligible patients were divided into the following categories: non-obstructive idiopathic asthenozoospermia $(<50 \%$ motile spermatozoa and normal concentration, $n=124)$ and oligoasthenozoospermia $\left(<20 \times 10^{6}\right.$ spermatozoa $\mathrm{ml}^{-1}$ and $<50 \%$ motile spermatozoa, $n=26)$. Azoospermic and oligozoospermic patients were excluded.

The control group consisted of 126 fertile men with normal semen parameters $\left(>20 \times 10^{6}\right.$ sperm $\mathrm{ml}^{-1},>40 \%$ progressive motile sperm, $>60 \%$ viable sperm, $>30 \%$ with normal morphology and $<1 \times 10^{6}$ white blood cells $\mathrm{ml}^{-1}$ ). All 126 fertile men recruited to serve as controls achieved normal fertilization after a series of diagnostic tests was performed on themselves and their partners. The males who were partners in infertile couples group, who exhibited normal semen parameters but unknown fertilization statuses, were not included in this study.
Semen samples were obtained from each individual participant in both the case and the control groups with informed consent, and genomic DNA was extracted from semen by a standard hydroxybenzene-chloroform method.

\section{Genetic analysis}

The sense and antisense primers used for amplifying the CAG-repeats in exon 1 of the $P O L G$ gene have been reported previously. ${ }^{21}$ The forward primer was fluorescently labeled with FAM (Carboxyfluorescein) at the $5^{\prime}$ end of the primer to analyze the PCR products in an automated DNA analyzer (ABI 3130; Applied Biosystems, Foster City, CA, USA). PCR for each sample was carried out in a $10-\mu \mathrm{l}$ reaction volume containing $10 \mathrm{ng}$ of DNA, $10 \mathrm{pmol} \mathrm{l}^{-1}$ of each primer, $200 \mathrm{mmol}^{-1} \mathrm{dNTPs}$ and $1 \times$ PCR buffer (TaKaRa, Dalian, China) containing $1.5 \mathrm{mmol} \mathrm{l}^{-1} \mathrm{MgCl}_{2}$ and $2 \mathrm{U}$ rTaq (TaKaRa). Amplification consisted of an initial denaturation step of 5 min at $94{ }^{\circ} \mathrm{C}$, followed by 30 cycles of $30 \mathrm{~s}$ denaturation at $94{ }^{\circ} \mathrm{C}, 30 \mathrm{~s}$ annealing at $65^{\circ} \mathrm{C}$, extension at $72{ }^{\circ} \mathrm{C}$ for $30 \mathrm{~s}$ and a final extension for 10 min at $72{ }^{\circ} \mathrm{C}$.

Electrophoresis of amplified products was conducted on an $\mathrm{ABI}$ 3130 DNA Analyzer (Applied Biosystems) for genotyping. A 5- $\mu \mathrm{l}$ volume of diluted PCR product (PCR product, $\mathrm{ddH}_{2} \mathrm{O} 1: 900$ ) was mixed with a $5-\mu l$ mixture of GS LIZ500 or GS 400HD size standard and Hi-Di formamide $(1: 100)$. The raw data were further analyzed using GeneMapper software (GeneMapper Software version 4.0; Applied Biosystems, 2005) to ascertain the length of the POLG-CAG allele. PCR and genotyping were repeated for all the samples to confirm the number of repeats. In addition, sequencing was used to define the size of the PCR product.

\section{Statistical analysis and meta-analysis}

Analysis of genetic data. The Hardy-Weinberg equilibrium (HWE) for the six Chinese Han populations was assessed by SHEsis (http:// analysis.bio-x.cn/myAnalysis.php). The differences among the six Chinese Han populations were calculated by a $\chi^{2}$ test using SPSS (version 17.0; SPSS Inc., Chicago, IL, USA). The difference in the number of CAG-repeats between the infertile and control groups was calculated by a $\chi^{2}$ test, which yielded a $P$ value and odds ratio (OR) with the corresponding 95\% confidence interval (CI), using SPSS. Statistical significance was defined as a $P$ value $<0.05$.

Statistics for meta-analysis. The results from this study and other casecontrol studies, $911-13,15-19$ were combined for meta-analysis. The departure from HWE for the control group in each study was assessed using a Pearson $\chi^{2}$ test for goodness of fit in the HWE program (http:// ihg2.helmholtz-muenchen.de/cgi-bin/hw/hwa1.pl). The meta-analysis was performed using STATA (version SE-10.1; Stata Corporation, College Station, TX, USA). The OR and 95\% CI were calculated to estimate differences in CAG-repeat lengths between cases and controls. On the basis of the individual OR, a pooled OR was estimated, from which the statistical significance was determined using a $Z$-test. The $\chi^{2}$-based $Q$-statistic test was used for the assessment of between-study heterogeneity. If $P<0.05$, then the heterogeneity was considered significant, indicating heterogeneity across studies. We used a random effects model (I-V heterogeneity) to combine the individual effect size estimates. ${ }^{21,22}$ Stratification analysis by ethnicity was conducted to estimate ethnicity-specific ORs. Sensitivity analysis was performed to assess the stability of these results. A single study involved in the meta-analysis was omitted each time to reflect the influence of the individual studies on the overall effect estimate. 
Table 1 Allele distribution by province and diagnosis in six Chinese samples

\begin{tabular}{lrrrrrrr}
\hline \multirow{2}{*}{ Group (2n) } & \multicolumn{8}{c}{ Allele distribution } \\
\cline { 2 - 8 } & 6 & 7 & 9 & $10(\%)$ & 11 & 12 & 13 \\
\hline Distribution by province & & & & & & & \\
General population (1070) & 1 & 2 & 0 & $1042(97.38)$ & 25 & 0 & 0 \\
$\quad$ Shangdong (258) & 1 & 0 & 0 & $254(98.45)$ & 3 & 0 & 0 \\
$\quad$ Guangxi (224) & 0 & 0 & 0 & $219(97.77)$ & 5 & 0 & 0 \\
$\quad$ Yunnan (262) & 0 & 2 & 0 & $255(97.33)$ & 5 & 0 & 0 \\
$\quad$ Hunan (154) & 0 & 0 & 0 & $148(96.10)$ & 6 & 0 & 0 \\
$\quad$ Guangdong (84) & 0 & 0 & 0 & $82(97.62)$ & 2 & 0 & 0 \\
$\quad$ Gansu (88) & 0 & 0 & 0 & $84(95.45)$ & 4 & 0 & 0 \\
Distribution by diagnosis & & & & & & & \\
Case (300) & 0 & 1 & 4 & $287(95.67)$ & 8 & 0 & 0 \\
$\quad$ Asthenozoospermia (248) & 0 & 0 & 4 & $236(95.16)$ & 7 & 0 & 0 \\
$\quad$ Oligoasthenozoospermia (52) & 0 & 0 & 0 & $51(98.00)$ & 1 & 0 & 0 \\
Control (252) & 0 & 0 & 2 & $245(97.22)$ & 3 & 1 & 1 \\
\hline
\end{tabular}

Table 2 Genotypes by province and diagnosis in six Chinese samples

\begin{tabular}{llllllllll}
\hline & \multicolumn{10}{c}{ Genotype } \\
\cline { 2 - 9 } Group (n) & $6 /$ & $7 /$ & $7 /$ & $9 /$ & $9 /$ & $10 / 10$ & $10 /$ & $10 /$ & $10 /$ \\
& 10 & 10 & 9 & 9 & 10 & $(\%)$ & 11 & 12 & 13 \\
\hline Distribution by province & & & & & & & & & \\
General population (535) & 1 & 2 & 0 & 0 & 0 & $507(94.77)$ & 25 & 0 & 0 \\
$\quad$ Shangdong (129) & 1 & 0 & 0 & 0 & 0 & $125(96.90)$ & 3 & 0 & 0 \\
$\quad$ Guangxi (112) & 0 & 0 & 0 & 0 & 0 & $107(95.54)$ & 5 & 0 & 0 \\
$\quad$ Yunan (131) & 0 & 2 & 0 & 0 & 0 & $124(94.66)$ & 5 & 0 & 0 \\
$\quad$ Hunan (77) & 0 & 0 & 0 & 0 & 0 & $71(92.31)$ & 6 & 0 & 0 \\
Guangdong (42) & 0 & 0 & 0 & 0 & 0 & $40(95.24)$ & 2 & 0 & 0 \\
$\quad$ Gansu (44) & 0 & 0 & 0 & 0 & 0 & $40(90.91)$ & 4 & 0 & 0 \\
Distribution by diagnosis & & & & & & & & & \\
Case (150) & 0 & 0 & 1 & 1 & 1 & $139(92.67)$ & 8 & 0 & 0 \\
$\quad$ Asthenozoospermia & 0 & 0 & 1 & 1 & 1 & $114(91.94)$ & 7 & 0 & 0 \\
$\quad(124)$ & & & & & & & & & \\
$\quad$ Oligoasthenozoospermia & 0 & 0 & 0 & 0 & 0 & $25(96.15)$ & 1 & 0 & 0 \\
$\quad$ 26) & & & & & & & & & \\
Control (126) & 0 & 0 & 0 & 0 & 2 & $119(94.44)$ & 3 & 1 & 1 \\
\hline
\end{tabular}

Potential publication bias was estimated by a funnel plot using Egger's linear regression test. $P<0.05$ was considered representative of statistically significant publication bias. $^{23}$

\section{RESULTS}

In the current study, we first determined the distribution of CAGrepeat length variation of the $P O L G$ gene in six Chinese Han populations from different provinces in China. The CAG-allele variant distribution satisfied HWE in all of the Chinese Han populations $(P>0.05)$. The 10-CAG-repeat length was the common allele, with an average frequency of $97.38 \%$, and there was no significant difference among the six Han populations (Table 1). The frequencies of the common genotype $(10 / 10)$ in these six groups were also similar (Table 2). Further, the CAG-repeat length variant in the POLG gene was analyzed in infertile patients with asthenozoospermia and oligoasthenozoospermia, as well as normozoospermic fertile men. The most frequently observed POLG-CAG allele in the case and control groups was the common 10-CAG-repeat allele, with frequencies of 95.67 and $97.22 \%$, respectively (Table 1 ). The most frequent genotype in the case and control groups was the homozygous wild type (10/10) in 92.67 and $94.44 \%$, respectively, followed by the heterozygous (10/ not 10) genotype in 6.00 and $5.56 \%$, respectively (Table 2). Interestingly, the homozygous mutant genotype (not 10/not 10) was found only in infertile men, with a frequency of $1.33 \%$. All these homozygous mutant genotype carriers were asthenozoospermic men. There was no notable difference of particular allele between the case and control groups $\left(\chi^{2}=0.75 ; P=0.38 ; 95 \%\right.$ CI: $0.58-4.01$; $\mathrm{OR}=1.53$ ).

Subsequently, we combined our data with data from nine published case-control studies involving a total of 2463 infertile men and 1480 fertile men in a meta-analysis to determine the association between the POLG-CAG-repeat variant and male infertility. The studies involved and their main characteristics are listed in Table 3. The genotype distributions of the CAG-repeat polymorphism were found to be in HWE in controls for all eligible studies, except the study by Rani et al. ${ }^{16}$ $(P<0.05)$. The OR and 95\% CI were used to assess the strength of the associations between the POLG-CAG allele (not 10 versus 10) (Figure 1) or genotype and the risk of male infertility in the homozygote comparison (not 10/not 10 versus 10/10), heterozygote comparison (10/not 10 versus 10/10), dominant model comparison (not $10 /$ not $10+10 /$ not 10 versus $10 / 10$ ) and recessive model comparison (not 10/not 10 versus 10/not 10+10/10) (Figure 2). Compared with the 10-CAG-repeat allele carriers, those without the 10-CAG-repeat had no significantly greater risk for male infertility (pooled $\mathrm{OR}=0.94$; 95\% CI: 0.60-1.48) under the random effects model. As shown in Figure 2, no overt main effects on male infertility risk were observed in all comparisons, and no obvious significance was observed among

Table 3 Characteristics of published studies included in this meta-analysis

\begin{tabular}{|c|c|c|c|c|c|c|c|c|c|c|c|c|c|}
\hline \multirow[b]{2}{*}{ Study } & \multirow[b]{2}{*}{ Year } & \multirow{2}{*}{$\begin{array}{l}\text { Participant } \\
\text { ethnicity }\end{array}$} & \multicolumn{5}{|c|}{ Case } & \multicolumn{5}{|c|}{ Control } & \multirow{2}{*}{$H W E$} \\
\hline & & & $n$ & Allele 10 & $10 / 10$ & 10/not 10 & $\begin{array}{l}\text { Not } 10 / \\
\text { not } 10\end{array}$ & $n$ & Allele 10 & $10 / 10$ & $10 /$ not 10 & $\begin{array}{l}\text { Not } 10 / \\
\text { not } 10\end{array}$ & \\
\hline $\operatorname{Rani}^{16}$ & 2008 & Indian & 509 & 850 & 382 & 105 & 22 & 241 & 407 & 182 & 44 & 15 & $3.85 \times 10^{-6}$ \\
\hline Plaseski ${ }^{15}$ & 2007 & Macedonian & 225 & 394 & 173 & 47 & 5 & 123 & 213 & 92 & 26 & 5 & 0.09 \\
\hline Amaral $^{12}$ & 2007 & Portuguese & 95 & 164 & 69 & 26 & 0 & 40 & 34 & 27 & 13 & 0 & 0.22 \\
\hline Harris $^{18}$ & 2006 & New Zealand & 182 & 309 & 134 & 41 & 7 & 93 & 147 & 57 & 33 & 3 & 0.50 \\
\hline $\mathrm{Yao}^{13}$ & 2006 & Chinese & 146 & 283 & 137 & 9 & 0 & 104 & 201 & 97 & 7 & 0 & 0.72 \\
\hline Aknin-Seifer ${ }^{17}$ & 2005 & Caucasian & 433 & 727 & 307 & 113 & 13 & 91 & 156 & 66 & 24 & 1 & 0.46 \\
\hline Jensen $^{11}$ & 2004 & Danish & 429 & 725 & 308 & 109 & 12 & 374 & 673 & 302 & 69 & 3 & 0.66 \\
\hline Krausz $^{19}$ & 2004 & Italian & 195 & 332 & 143 & 47 & 5 & 190 & 308 & 126 & 58 & 6 & 0.83 \\
\hline Rovio $^{9}$ & 2001 & Finnish and English & 99 & 145 & 55 & 35 & 9 & 98 & 178 & 80 & 18 & 0 & 0.32 \\
\hline $\begin{array}{l}\text { The present } \\
\text { study }\end{array}$ & 2010 & Chinese & 150 & 287 & 139 & 9 & 2 & 126 & 245 & 119 & 7 & 0 & 0.75 \\
\hline
\end{tabular}

Abbreviation: HWE, Hardy-Weinberg equilibrium. 


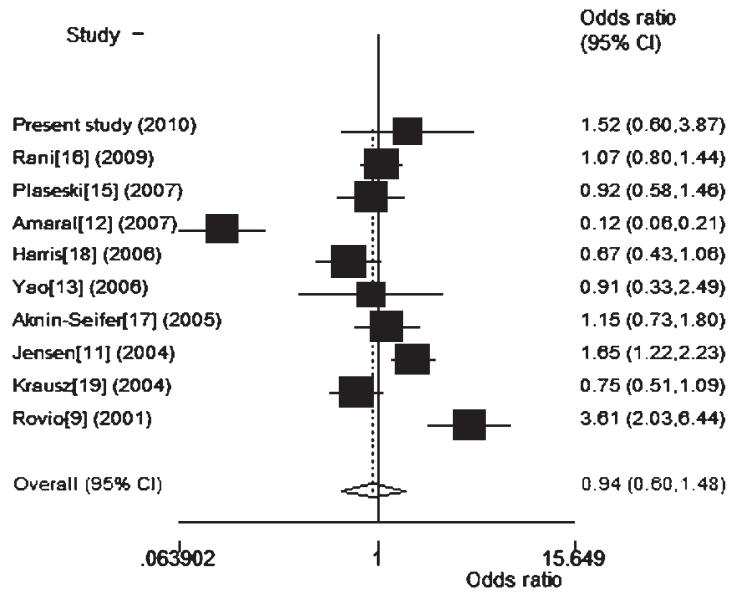

Figure 1 Meta-analysis with a random effects model for the ORs of male infertility risk associated with CAG-repeat variant alleles (not 10 versus 10 ). $\mathrm{Cl}$, confidence interval; OR, odds ratio.
\% Weight

7.8

11.3

10.5

9.7

10.8

7.4

10.8

11.2

10.9

9.9

the following comparisons: homozygote comparison (not 10/not 10 versus 10/10: $\mathrm{OR}=1.34$; 95\% CI: 0.66-2.72) (Figure $2 \mathrm{~b}$ ), heterozygote comparison (10/not 10 versus 10/10: OR=1.04; 95\% CI: 0.78 1.38) (Figure 2a), dominant model comparison (not 10/not $10+10 /$ not 10 versus 10/10: $\mathrm{OR}=1.08$; 95\% CI: $0.79-1.47$ ) (Figure 2c) and recessive genetic comparison (not 10/not 10 versus 10/not 10+10/10: $\mathrm{OR}=1.31$; 95\% CI: 0.68-2.55) (Figure 2d). There was notable heterogeneity for the allele $\left(\chi^{2}=84.16\right.$; degree of freedom (d.f.) $=9$, $P_{\text {heterogeneity }}=0.000, I^{2}=88 \%$ ) (Figure 1$)$, heterozygote $\left(\chi^{2}=23.02\right.$, d.f. $\left.=9, \quad P_{\text {heterogeneity }}=0.006, \quad I^{2}=61 \%\right)$ and dominant model $\left(\chi^{2}=29.79\right.$, d.f. $\left.=9, P_{\text {heterogeneity }}=0.000, I^{2}=70 \%\right)$ comparisons, but not for the homozygote and recessive model comparison $\left(\chi^{2}=13.58\right.$, d.f. $=7, \quad P_{\text {heterogeneity }}=0.059, \quad I^{2}=48 \% \quad$ and $\quad \chi^{2}=12.25 ; \quad$ d.f. $=7$; $P_{\text {heterogeneity }}=0.093 ; I^{2}=43 \%$ ) (Figure 2 ). We then evaluated the source of heterogeneity in these comparisons by ethnic stratification. We observed that heterogeneity still existed in European subethnicities (Table 4). The sensitivity analysis was performed by deleting a single study involved in the meta-analysis each time to reflect the influence of the individual result on the pooled ORs (Figure 3).

a

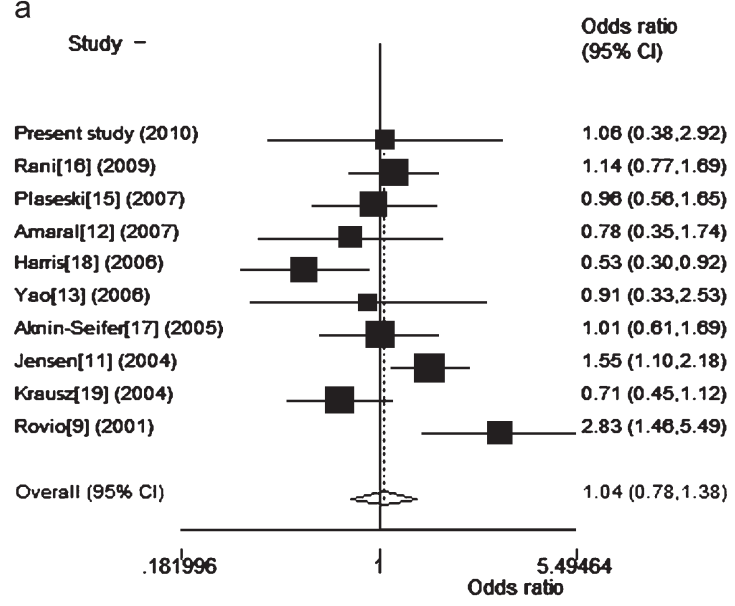

C

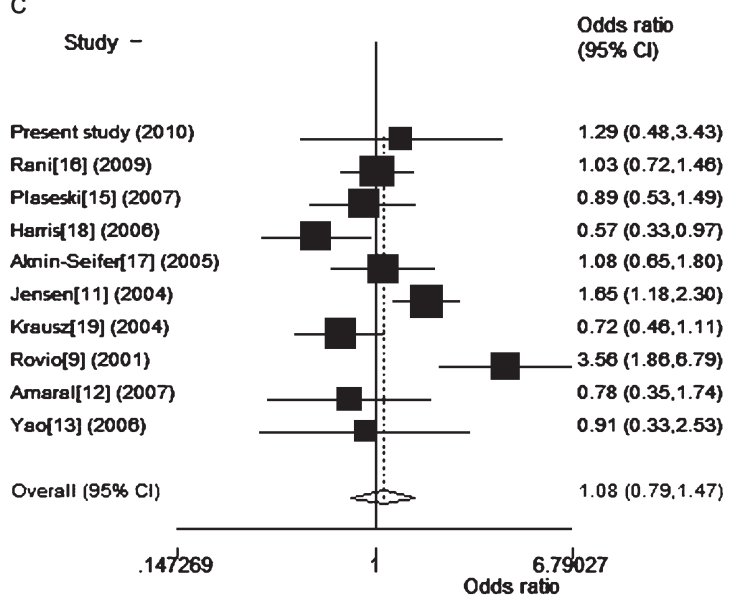

b

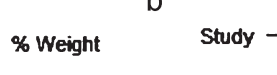

5.4

Present study (2010)

Rani[18] (2009)

Plaseski[15] (2007)

Harris[18] (2008)

Aknin-Seifer 17] (2005)

Jensen[11] (2004)

Krausz 19] (2004)

Rovio[9] (2001)

Amaral[12] (2007)

Yso[13] (2008)

Overall (95\% Cl)

$\begin{array}{ccc}.002069 \quad 1 \quad \text { Odds ratio } & 488\end{array}$

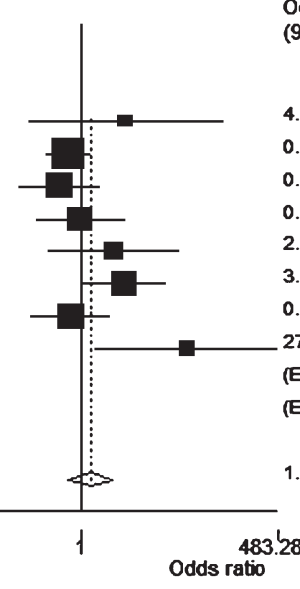

(95\% Cl)

\% Weight

$4.11(0.20 .88 .38)$

$0.70(0.35 .1 .38)$

$0.53(0.15 .1 .88)$

$0.99(0.25 .3 .98)$

$2.79(0.36 .21 .74)$

$3.92(1.10 .14 .04)$

$0.73(0.22 .2 .46)$

$27.56(1.57 .483 .29)$

(Excluded)

(Excluded)

$1.34(0.68 .2 .72)$

\% Weight Study -

Odds ratio

(95\% Cl)

\% Weight

Present study (2010)

Rani[16] (2009)

Plaseski[15] (2007)

Harris[18] (2008)

Aknin-Seifer[17] (2005)

Jensen[11] (2004)

Krausz [19] (2004)

Rovio[9] (2001)

Amaral[12] (2007)

YaO[13] (2008)

Overall (95\% Cl)

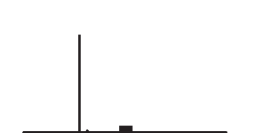

$4.09(0.19 .86 .05)$

$0.88(0.35 .1 .34)$

$0.54(0.15 .1 .89)$

$1.20(0.30 .4 .75)$

$2.79(0.36 .21 .57)$

$3.56(1.00 .12 .71)$

$0.81(0.24 .2 .69)$

20.68 (1.19.360.41)

(Excluded)

(Excluded)

$1.31(0.68 .2 .55)$
4.5

22.9

15.0

13.7

8.4

14.9

15.6

5.0

0.0

0.0

.002775

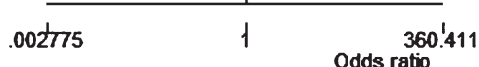

Figure 2 Pooled odds ratios of male infertility by different genetic model comparisons. (a) The heterozygote comparison (10/not 10 versus 10/10); (b) the homozygote comparison (not 10/not 10 versus 10/10); (c) the dominant model comparison (not 10/not 10+10/not 10 versus 10/10); (d) the recessive genetic comparison (not 10/ not 10 versus 10/not 10+10/10). $\mathrm{Cl}$, confidence interval. 


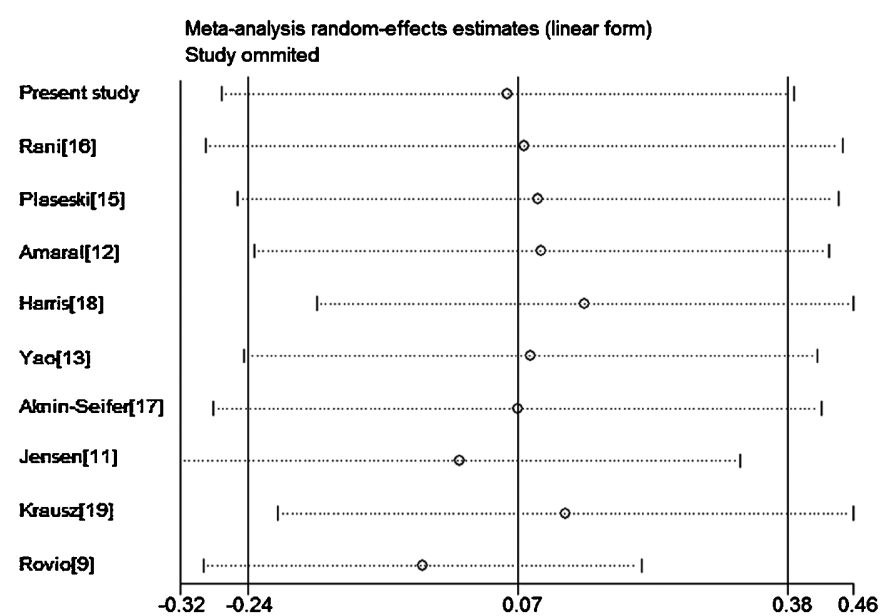

Figure 3 Sensitivity analysis of this meta-analysis. The vertical axis indicates the log odds ratio of the POLG-CAG-repeat variant that has been estimated. The meta-analysis is dominated by the Rovio et al.'s study; ${ }^{9}$ hence omission of other studies makes little or no difference. If the observation made by Rovio et al. is omitted, then there appears a clear result that the POLG-CAG-repeat variant is not associated with male infertility. POLG, polymerase $\gamma$.

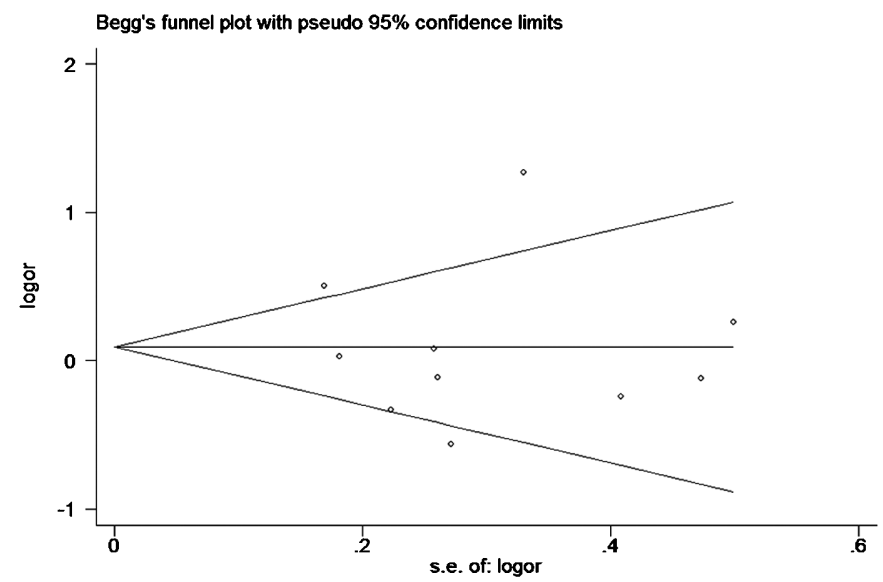

Figure 4 Funnel plot for publication bias of the meta-analysis of male infertility risk and CAG-repeat variant of POLG gene (dominant model comparison: not 10/ not $10+10 /$ not 10 versus 10/10). POLG, polymerase $\gamma$.

Results indicated that the studies by Rovio et al., ${ }^{9}$ Jensen et al. ${ }^{11}$ and Harris et al. ${ }^{18}$ were the main origins of heterogeneity, especially the study by Rovio et al.. ${ }^{9}$ The heterogeneity changed markedly when this study was omitted (heterozygote comparison: $\mathrm{OR}=0.96,95 \% \mathrm{CI}$ : $0.75-1.22, \chi^{2}=14.42$, d.f. $=8, P_{\text {heterogeneity }}=0.071, I^{2}=45 \%$; dominant model: $\quad$ OR $=0.96, \quad 95 \%$ CI: $0.75-1.24, \quad \chi^{2}=16.26$, d.f. $=8$, $\left.P_{\text {heterogeneity }}=0.039, I^{2}=51 \%\right)$. Begg's funnel plot and Egger's test were performed to assess publication bias. ${ }^{23}$ The shape of the funnel plot revealed no publication bias for all meta-associations (Figure 4).

\section{DISCUSSION}

In the present study, there was no difference in the distribution of the POLG polymorphism among six general samples of healthy Chinese Han people living in six different provinces. Previous studies have documented the distribution of the POLG-CAG-repeat length variant in different populations around the world. We performed pairwise comparisons of previously published data from different populations (Finnish, English, German, Danish, Indian, Dutch, African, North 
Table 5 Previous studies on the frequencies of each CAG-repeat genotype

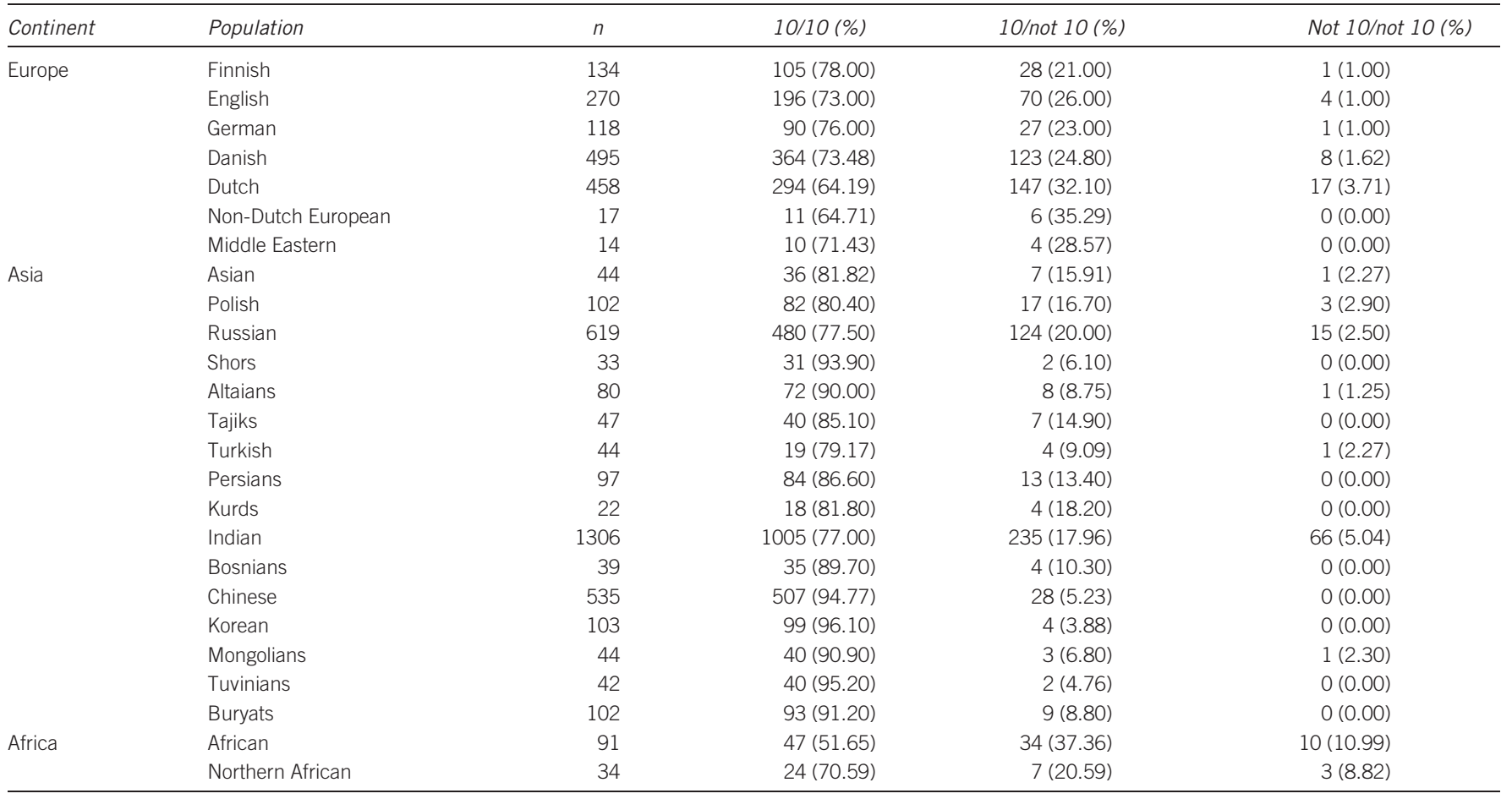

African, Turkish, non-Dutch European, Middle Eastern, Asian, Korean, Mongolian, Shor, Altaian, Tuvinian, Buryat, Tajik, Persian, Kurd, Bosnian, Polish and Russian) on the frequency of the CAGrepeat polymorphisms (Table 5). ${ }^{10,14,19,24}$ We found differences in the frequencies of the three POLG-CAG-repeat genotypes between geographically and ethnically related populations. The prevalence of homozygous wild type (10/10) was shown to be exceedingly high in Chinese (97.38\%) and Koreans (96.1\%), but low in Africans (51.65\%). Overall, the frequencies of all CAG-repeat genotypes were similar for groups from the same continent (Table 5 and Supplementary Table 1), but differed when comparing groups from different continents. These results indicate that there is a significant difference in the frequencies of the three CAG-repeat genotypes between geographically and ethnically related populations. Therefore, an assessment of the study population's ethnic and genetic background is needed before an association study can be performed.

In this study, to determine the association between the POLG-CAGrepeat polymorphism and male infertility in a Chinese population, we compared infertile men (asthenozoospermia and oligoasthenozoospermia) with normozoospermic fertile controls. Our study revealed that the risk of infertility is slightly higher for males carrying the not 10/not 10 genotype according to a different genetic comparison model (Figure 2); however, our results did not show any statistical difference between the case and control groups, which indicated that the POLGCAG-repeat might not be a susceptibility locus for male infertility in the Chinese people.

Many studies have investigated the association between male infertility and the trinucleotide CAG-repeat polymorphism in the POLG gene, but the results are widely divergent. To obtain a more complete and precise estimate of the association, we collected all available data to perform a comprehensive meta-analysis. A prior meta-analysis of six published studies suggested that there was no association between the POLG-CAG-repeat polymorphism and male infertility. ${ }^{25}$ However, several studies published in recent years were not included in the previous meta-analysis. Therefore, we combined our results with those of nine published studies and conducted a comprehensive meta-analysis with the goals of providing more reliable findings on the significance of the association and quantitatively investigating the sources of heterogeneity between the studies. We performed our meta-analysis based on a different model of comparisons and found that the POLG-CAG-repeat variant was not associated with male infertility.

Heterogeneity testing detected conspicuous heterogeneity among the studies included in our meta-analysis. Even when we stratified the studies by the ethnicity of their participants, heterogeneity was still detected in European subgroups. The ethnic difference might be one of the causes of the heterogeneity. Our results show that the allele frequencies of the POLG-CAG-repeat variants and genotype frequencies vary across different ethnic groups. The variability of the association might be influenced by the different frequencies of this polymorphism in populations of different ethnicities. As evidenced by a recent data mining study, differences in allele frequencies between groups can result in a reversal of allelic effects. ${ }^{26}$ Another cause for the discrepancy might be that this polymorphism has a different role in individuals of different ethnicities. There might be different genetic modifiers among some of the populations studied or some other functional polymorphisms that interact with this CAG-repeat. The POLG-CAG-repeat variant may not, by itself, exhibit a significant association with male infertility in all or most studies because its effect may be small and dependent on genotypes at other loci that compensate for variation in the locus under study. Furthermore, the heterogeneity might be caused by the different case criteria employed by the published studies. Some studies combined three (azoospermia, oligozoospermia and asthenozoospermia) or more subgroups of idiopathic male infertility to examine the distribution of CAG-repeat variants of the POLG gene. ${ }^{12,13,15,17-19}$ On the contrary, some studies analyzed only one subgroup or excluded some subgroups, such as Rani et al., ${ }^{16}$ who studied only the association between the CAG-repeat variant and 
oligoasthenozoospermia; Rovio et al. ${ }^{9}$ who excluded azoospermic and severely oligozoospermic men; and this study, which only included asthenozoospermic and oligoasthenozoospermic men. All studies excluded patients with genetic causes of infertility (karyotype mutations, Kallman syndrome, Y-chromosome microdeletions and cystic fibrosis mutations) from the analysis; however, not all known genetic causes of male infertility were reported to be excluded in all studies. It is possible that the distribution of this polymorphism among different male infertility subcategories is quite different. However, these studies combined the data on males in different subcategories, and the original data are not available for a stratification analysis.

In conclusion, we suggest that the frequencies of the CAG-repeat variants in the $P O L G$ gene are significantly different in diverse ethnic groups and that this polymorphism is not associated with male infertility among Chinese people. Through a meta-analysis using several types of comparison models, we demonstrated that there is no manifest association between the POLG-CAG-repeat variant and male infertility. Susceptibility to male infertility may be confined to a certain population. There is no need to carry out further studies on the distribution of the POLG-CAG-repeat variant in the context of male infertility. It would be more productive to look for other interesting genetic factors or discuss the interaction of the POLG-CAG-repeat variant with other polymorphisms. Large, well-designed studies are warranted to validate the association in specific ethnic populations.

Note: Supplementary information is available on the Asian Journal of Andrology website (http://www.nature.com/aja/).

\section{AUTHOR CONTRIBUTIONS}

J.Y.C., Y.F.Y. and C.J.Z. conceived and designed the experiments. S.Y.L. and L.S. performed the genetic analysis. S.Y.L., Y.F.Y., J.B.C. and L.S. analyzed the data. H.Y.P., S.Y.L. and C.J.Z. conducted the clinic andrological examinations. H.Y.P., K.Q.L., L.Y., X.Q.H. and H.S. performed the sample collection. S.Y.L. and J.Y.C. drafted the paper.

\section{COMPETING FINANCIAL INTERESTS}

The authors declare that they have no competing financial interests.

\section{ACKNOWLEDGMENTS}

This work was supported by the China Medical Board of New York Inc. (No. 04-805).

1 Poongothai J, Gopenath TS, Manonayaki S. Genetics of human male infertility. Singapore Med J 2009; 50: 336-47.

2 O'Flynn OB, Katherine L, Varghese AC, Agarwal A. The genetic causes of male factor infertility: a review. Fertil Steril 2010; 93: 1-12.
3 Carra E, Sangiorgi D, Gattuccio F, Rinaldi AM. Male infertility and mitochondrial DNA. Biochem Biophys Res Commun 2004; 322: 333-9.

4 St John JC, Jokhi RP, Barratt CL. The impact of mitochondrial genetics on male infertility. Int J Androl 2005; 28: 65-73.

5 Longley MJ, Graziewicz MA, Bienstock RJ, Copeland WC. Consequences of mutations in human DNA polymerase gamma. Gene 2005; 354: 125-31.

6 Chan S, Copeland WC. DNA polymerase gamma and mitochondrial disease: understanding the consequence of POLG mutations. Biochim Biophys Acta 2009; 1787: 312-9.

7 Hudson G, Chinnery PF. Mitochondrial DNA polymerase-gamma and human disease. Hum Mol Genet 2006; 15 Spec No 2: R244-52.

8 Ropp PA, Copeland WC. Cloning and characterization of the human mitochondrial DNA polymerase, DNA polymerase gamma. Genomics 1996; 36: 449-58.

9 Rovio AT, Marchington DR, Donat S, Schuppe HC, Abel J et al. Mutations at the mitochondrial DNA polymerase $(P O L G)$ locus associated with male infertility. Nat Genet 2001; 29: 261-2.

10 Malyarchuk BA, Papuga M, Grzybowski T, Rogozin IB, Wozniak M et al. Low variability of the POLG (CAG) n repeat in north Eurasian populations. Hum Biol2005; 77: 35566.

11 Jensen $M$, Leffers $H$, Petersen JH, Nyboe AA, Jorgensen $N$ et al. Frequent polymorphism of the mitochondrial DNA polymerase gamma gene (POLG) in patients with normal spermiograms and unexplained subfertility. Hum Reprod 2004; 19: 65-70.

12 Amaral A, Ramalho-Santos J, St John JC. The expression of polymerase gamma and mitochondrial transcription factor $A$ and the regulation of mitochondrial DNA content in mature human sperm. Hum Reprod 2007; 22: 1585.

13 Yao N, Zheng JF, Peng YF, Li ZX, Peng ZQ et al. CAG repeats of DNA polymerase gamma in Chinese males and relationship of idiopathic male infertility to CAG repeats. Zhonghua Nan Ke Xue 2006; 12: 681-4, 688. Chinese.

14 Westerveld GH, Kaaij-Visser L, Tanck M, van der Veen F, Repping S. CAG repeat length variation in the polymerase gamma (POLG) gene: effect on semen quality. Mol Hum Reprod 2008; 14: 245.

15 Plaseski T, Noveski P, Dimitrovski C, Efremov GD, Plaseska-Karanfilska D. CAG repeat polymorphism of the mitochondrial DNA polymerase gamma gene in macedonian infertile and fertile men. Balkan J Med Genet 2007; 10: 37-42.

16 Rani DS, Carlus SJ, Poongothai J, Jyothi A, Pavani K et al. CAG repeat variation in the mtDNA polymerase gamma is not associated with oligoasthenozoospermia. Int $J$ Androl 2009; 32: 647-55.

17 Aknin-Seifer IE, Touraine RL, Lejeune H, Jimenez C, Chouteau J et al. Is the CAG repeat of mitochondrial DNA polymerase gamma $(P O L G)$ associated with male infertility? A multi-centre French study. Hum Reprod 2005; 20: 736-40.

18 Harris TP, Gomas KP, Weir F, Holyoake AJ, McHugh P et al. Molecular analysis of polymerase gamma gene and mitochondrial polymorphism in fertile and subfertile men. Int J Androl 2006; 29: 421-33.

19 Krausz C, Guarducci E, Becherini L, Degl'Innocenti S, Gerace L et al. The clinical significance of the POLG gene polymorphism in male infertility. J Clin Endocrinol Metab 2004; 89: 4292-7.

20 World Health Organization. Laboratory manual for the examination of human semen and sperm-cervical mucus interaction. 4th ed. New York: Cambridge University Press; 1999.

21 Higgins JP, SG, Thompson JJ, Deeks Altman DG et al. Measuring inconsistency in meta-analyses. BMJ 2003; 327: 557-60.

22 Higgins JP, Thompson SG. Quantifying heterogeneity in a meta-analysis. Stat Med 2002; 21: 1539-58.

23 Egger M, Davey SG, Schneider M, Minder C. Bias in meta-analysis detected by a simple, graphical test. BMJ 1997; 315: 629-34.

24 Rovio AT, Abel J, Ahola AL, Andres AM, Bertranpetit J et al. A prevalent POLG CAG microsatellite length allele in humans and African great apes. Mamm Genome 2004; 15: 492-502

25 Tuttelmann F, Rajpert-De Meyts E, Nieschlag E, Simoni M. Gene polymorphisms and male infertility a meta-analysis and literature review. Reprod Biomed Online 2007; 15: 643-58.

26 Greene CS, Penrod NM, Williams SM, Moore JH. Failure to replicate a genetic association may provide important clues about genetic architecture. PLoS One 2009; 4: e5639. 